\title{
PATTERN-ORIENTED INVERSE SIMULATION FOR ANALYZING SOCIAL PROBLEMS: FAMILY STRATEGIES IN CIVIL SERVICE EXAMINATION IN IMPERIAL CHINA
}

\author{
CHAO YANG \\ College of Information Science and Engineering, Hunan University \\ Changsha, Hunan 410082, China \\ Department of Computational Intelligence and Systems Science, Tokyo Institute of Technology \\ Yokohama, Kanagawa 226-8503, Japan \\ yangchao@trn.dis.titech.ac.jp \\ SETSUYA KURAHASHI \\ Graduate School of Business Sciences, University of Tsukuba \\ Bunkyo-ku, Tokyo 112-0012, Japan \\ kurahashi@gssm.otsuka.tsukuba.ac.jp \\ ISAO ONO \\ Department of Computational Intelligence and Systems Science, Tokyo Institute of Technology \\ Yokohama, Kanagawa 226-8503, Japan \\ isao@dis.titech.ac.jp \\ TAKAO TERANO \\ Department of Computational Intelligence and Systems Science, Tokyo Institute of Technology \\ Yokohama, Kanagawa 226-8503, Japan \\ terano@dis.titech.ac.jp \\ Received (received date) \\ Revised (revised date)
}

\begin{abstract}
This paper proposes an agent-based model to investigate the role of parental relationships and intergenerational reproduction of cultural capital to understand the long-term professional success of an elite family line during the Ming and Qing dynasties in imperial China. We implemented the model by a new method: the pattern-oriented inverse simulation (POIS) method, where multiple patterns observed in an elite family line are employed to guide the model design and test alternative assumptions as family strategies. A genetic algorithm(GA) based inverse technique is applied to fit the simulation outputs with actual data grouped in time intervals as patterns. The simulation results discovered family strategies sustained by the examination systems in imperial China, which relates to important sociological theories on the impact of the intergenerational reproduction of cultural capital within family circles on social inequality in the individual entry in labor and professional markets. The case study also proved that the new methodology of POIS can improve our current practices for systematically exploring simulation parameter space and fit model output with actual data.
\end{abstract}

Keywords: pattern-oriented modeling; inverse simulation; agent-based complex systems; 
pattern-oriented inverse simulation; social problems.

\section{Introduction}

In the methodology of study of agent-based complex systems (ACSs),PatternOriented Modeling (POM)has been suggested as a unifying framework for decoding the internal organization of ACSs [6], which attempts to make bottom-up modeling such as cellular automata and agent-based modelings more rigorous and comprehensive. POM assumes that multiple patterns observed in a real system characterize the system and its dynamics, and often indicate the essential information underlying observed patterns to address real problems.

Grimm et al. gave the definition of patterns as "observations of any kind showing non-random structure and therefore containing information on the mechanisms from which they emerge" [6]. They discussed how observed patterns could be used in POM to find the internal organization of ACSs through three key points: (1) guide the design of model structure, for example, in [13], they used horizontal and vertical patterns and canopy gaps pattern to guide the modeling of spatio-temporal dynamics of beech forests of central Europe; (2) test and contrast theories for agent behavior, for instance, two theories of the herdsmen's reasoning were contrasted to explore what determined the access of herdsmen to pasture lands [14]; and (3) reduce parameter uncertainty, for example, five observed patterns used as filters resulted in only 10 of the 557 parameter sets reproduced out of all of them [20].

The POM strategy has been widely used in ecology [13, 14, 20], but up until now not so many models have been used for social problems[7]. This paper studies such a topic, by using the POM strategy to analyze real social systems.

Inverse Simulation (IS) is a method, which employs optimization algorithms such as genetic algorithms (GAs) to fit all micro-level parameters by finding values to reproduce macro-level parameters or patterns, through which real problems can be addressed[18]. It executes simulation steps in a reverse order: (1) a systemlevel objective function is set, (2) the simulated worlds are then evolved to fit to macro-level parameters or patterns, and (3) the micro-level agent parameters are optimized by means of the objective function. As for the objective function, usually sum-of-squares errors between observed values (macro-level parameters or patterns) from a real system and experimental values that are calculated by performing the simulation are used [18]. The key idea of IS is to find optimal parameter values by means of solving "inverse problems" [16, 17].

Terano and Kurahashi employed IS to historical and cultural anthropology in imperial China [9]. They successfully reproduced the successful examination candidate pattern of a family line and showed an applicability of IS with a single pattern to historical studies [9]. Their results discovered a positive influence from artistic capital to knowledge capital while the inverse effect is small. In imperial China, there were cultural activities which provided chances for appreciating drawing and poems. For example, the Y family line had a tradition of being outstanding at draw- 
ing. Grandfathers always bring their grandsons to attend such cultural activities, in order to find chance to talk and learn the official languages from those families who were literate in classical Chinese. Through such a special life style, cultural capital reproduction and family fortunes could be maintained.

Following their work, we reexamined the issue with an enlarged parameter space by using IS with macro-level parameters, implemented by GA on a grid environment [21]. The optimal model structure with its parameter values discovered the women's role of mothers and aunts from a reciprocal family relationship in educating children [21]. However, our previous work ignored the importance of using observed multiple patterns to guide the agent-based modeling for analyzing family strategies. We failed to reproduce multiple observed patterns of the $\mathrm{Y}$ family line $[9,21]$.

This paper proposes a new methodology, Pattern-Oriented Inverse Simulation (POIS) to improve our current practices for systematically exploring simulation parameter space and fit model output with actual data. To demonstrate the practical applicability of POIS for analyzing social problems, we applied POIS to a historical simulation domain. The parameters values obtained by the previous simulation models failed to reproduce different types of observed patterns of the real family system. This study makes use of multiple observed patterns to guide the model design and test alternative assumptions as family strategies. Here, pattern refers to actual data grouped in time intervals. The evaluation sets how well the simulation output fit with the actual data. We also emphasize that the model focused on family strategies(norms) behind the whole family system in conveying cultural capitals to the descendants, rather than individual-based decision making.

The remaining part of this paper is organized as follows: Section 2 introduces the examination system and its important position in imperial China. Section 3 explains the genealogical records in China and discusses multiple patterns observed in the Y family line. Section 4 describes the agent model by following ODD protocol. Section 5 provides the implementation of agent model by POIS. Section 6 gives the simulation experiments and Section 7 discusses the results. Finally, Section 8 gives concluding remarks and an outlook on future work.

\section{Examination System in Imperial China}

In imperial China, there were imperial civil service examinations that served as the basic of a selection system for the recruitment of government officials. The examination system in imperial China comprised four levels of examinations: a qualifying examination, a provincial examination, a metropolitan examination and a palace examination. But first of all, with a preliminary step, candidates first took a county/department/prefectural licensing examination where those who passed were bestowed with Shengyuans (the status licentiate). Next, Shengyuan took a county/ prefectural/town qualifying examination and then, if they passed, they were called Gongsheng(tribute student) or Jiansheng(state student). Gongsheng and Jiansheng who passed the provincial examination, the metropolitan exami- 
nation received Juren, Gongshi(tribute literatus) degrees. At the highest level of this examination system, of those who passed and placed in the top three of the metropolitan examination could go on to take the palace examination. If successful, they would be recognized as Jinshi(literatus presented to emperor for appointment). Thus, Jinshi, Gongshi, Juren, Gongsheng, Jiansheng, and Shengyuan refer to highest, second highest, etc. degree of successful candidates in the examination systems.

Even though only a small fraction of those who attempted the examinations passed them and received titles, every year over millions of people from all levels of society attempted them and regarded the system as the most authoritative method for achieving their own personal success through passing such subsequent examinations. In each year, only 100 out of 100,000 people passed the final examination.

However, it took a huge investment in time, effort, and training in order to achieve such success. Such candidates were set by their family, clan, and lineage as the strategic targets of the social reproduction of their community. Each house is responsible to obtain and maintain these elite positions at the initial stage of educating a son. As Elman pointed out that it is possible for seemingly ordinary candidates to have achieved academic success because they had bureaucrats among their close relatives or affinities to the same lineage [3], which indicated the advantages for the families in having successful candidates. That is, candidates who came from a family which had the tradition of learning classics and spoke the official language gained superiority for a successful future social and political career.

The examination system was finally abolished in 1905 as(1)the spread-out official corruption made individuals can buy their examination qualifies by casting money, and the elimination of the value of the examination system; (2)the inability of the imperial examination system for industrial development, such as engineering, etc.

\section{Multiple Patterns of the Y Family Line}

In China, records of family trees and lineage had been made from old times and kept as genealogical records. This is known as Zupu. It was a paternal record from the primogenitor, including the blood relationship and each member's profile, occupation, qualified status of the examinations, achievement, art, contribution, qualified status of the examinations of wife's birth family and/or daughter's married family.

In this study, we use the genealogical records of the Y family in the Ming and Qing Dynasties to discover family strategies in order to sustain success with the examination systems in imperial China. The $\mathrm{Y}$ family is one of the typical cases, which continued to produce successful candidates. This indicates that the family strategies may have made for an advantage on these examinations, and that culture and habits are actually invisible standards for selection.

Following our previous work, we prepared two types of matrix to formulate the genealogical records in a convenient way for use in analysis and simulation. The genealogical records of the $\mathrm{Y}$ family line contained data for a total of 1,237 persons. 
We then prepared an adjacency matrix of $1237 \times 1237$ to show the relationship between fathers and sons, and "0, 1" represents this relationship. As shown in Fig.1, if a person $i$ has a son $j$, the element $i j$ of the adjacency matrix is 1 , otherwise 0 .

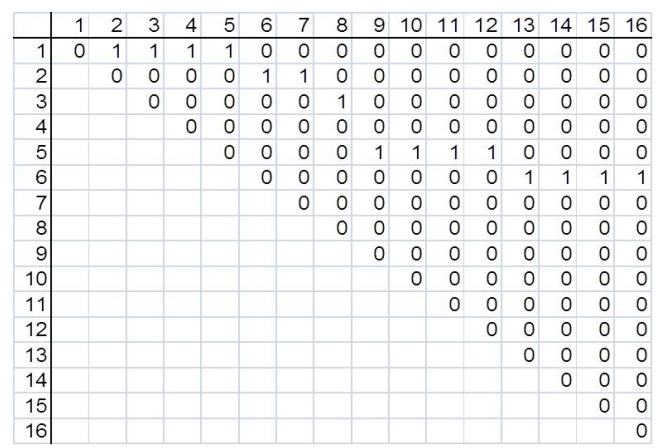

Fig. 1. The adjacency matrix of the Y family (Part).

In the same manner, we prepared the attribute matrix of each person from profile data in the genealogical records (Fig.2).

\begin{tabular}{|r|r|r|r|r|r|r|r|r|r|r|r|}
\hline & jinshi & gongshi & juren & gongsheng & jiansheng & shengyuan & painter & birth year & wife daughter \\
\hline 1 & 0 & 0 & 0 & 0 & 0 & 0 & 0 & 1468 & 0 & 0 \\
\hline 2 & 0 & 0 & 0 & 0 & 0 & 0.5 & 0 & 1492 & 0 & 0 \\
\hline 3 & 0 & 0 & 0 & 0 & 0 & 0 & 0 & 1492 & 0 & 0 \\
\hline 4 & 0 & 0 & 0 & 0 & 0 & 0 & 0 & 1492 & 0 & 0 \\
\hline 5 & 0 & 0 & 0 & 0 & 0 & 0 & 0 & 1492 & 0 & 0 \\
\hline 6 & 0.5 & 0 & 0 & 0 & 0 & 0 & 0 & 1518 & 0 & 0 \\
\hline 7 & 0 & 0 & 0 & 0 & 0 & 0.5 & 0 & 1522 & 0 & 0 \\
\hline 8 & 0 & 0 & 0 & 0 & 0 & 0 & 0 & 1522 & 0 & 0 \\
\hline 9 & 0 & 0 & 0 & 0 & 0 & 0 & 0 & 1522 & 0 & 0 \\
\hline 10 & 0 & 0 & 0 & 0 & 0 & 0 & 0 & 1541 & 0 & 0 \\
\hline 11 & 0 & 0 & 0 & 0 & 0 & 0 & 0 & 1545 & 0 & 0 \\
\hline 12 & 0 & 0 & 0 & 0 & 0 & 0 & 0 & 1548 & 0 & 0 \\
\hline 13 & 0 & 0 & 0 & 0 & 0 & 0.5 & 0 & 1544 & 0.5 & 0 \\
\hline 14 & 0 & 0 & 0 & 0 & 0 & 0.5 & 0 & 1548 & 0 & 0.5 \\
\hline 15 & 0 & 0 & 0 & 0 & 0 & 0 & 0 & 1550 & 0.5 & 0.5 \\
\hline 16 & 0 & 0 & 0 & 0 & 0 & 0 & 0 & 1550 & 0 & 0 \\
\hline
\end{tabular}

Fig. 2. The attribute matrix of the Y family (Part).

As shown in Fig.2, the attributes involve received titles from the examinations, painter, poet, merchant, birth year, qualified status of the examinations of the wife's birth family and the daughter's married family. And among these elements, the attribute of qualified status of the examination (Jinshi, Gongshi, Juren, Gongsheng, Jiansheng and Shengyuan), and the attribute of individual's expertise in painting, 
poetry, or his philanthropic acts (painter, poet, and merchant) are represented by "0, 0.5". For example, if a person $i$ has a qualified title of a successful candidate and the title ID is $k$, then the element $i k$ of the attribute matrix is 0.5 , otherwise 0 . Especially, if a person married a women and/or his daughter married a man with the father received qualified titles at the examinations, then the attribute of his wife and/or his daughter is represented by 0.5 , or else is 0 . Their birth years are represented by four-figure numbers.

Each member has one candidate status for the examination system and also has multiple statuses of his occupation, art, his wife's birth family and his daughter's married family. Following the relationship and attributes in the above mentioned two matrices, we constructed the $\mathrm{Y}$ family network. Fig.3 shows parts of the network of the $\mathrm{Y}$ family. As shown in Fig.3, each individual is represented as a node, with its label consistent with the individual identification (integers from 1 to 1237)described in the adjacency matrix. The links between two nodes represent relationship between fathers and sons, introduced by the "1" values of the adjacency matrix. The successful degree of individuals in the examinations or painters are denoted by nodes filled with colors, where red nodes represent successful candidates who received titles as Jinshi, Gongshi, or Juren; green nodes are successful candidates who received titles as Gongsheng, Jiansheng and Shengyuan; blue nodes are painters, and the remaining nodes are neither successful candidates nor painters, marked as black. A new family image becomes clear as a recognized network.

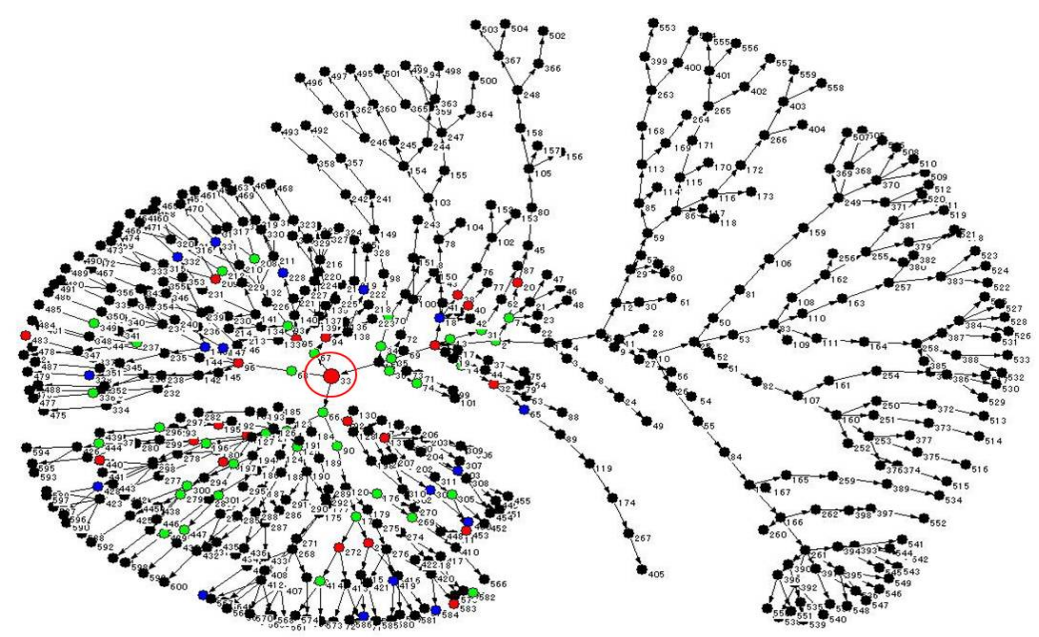

Fig. 3. The Network of the Y Family Line (Part).

An interesting phenomenon is that, even in such a so-called elite family line, a seventy percent candidate success rate is concentrated on the branch of node 
No.33(which was the ancestor of the successful branch,marked by the red circle) while all the other branches hold only a thirty percent success rate. By grouping the actual data in time intervals of ten years period from the individuals' birth years, we obtained successful candidate patterns at different scales (Fig.4) and, successful candidate pattern and painter pattern with different types(Fig.5).
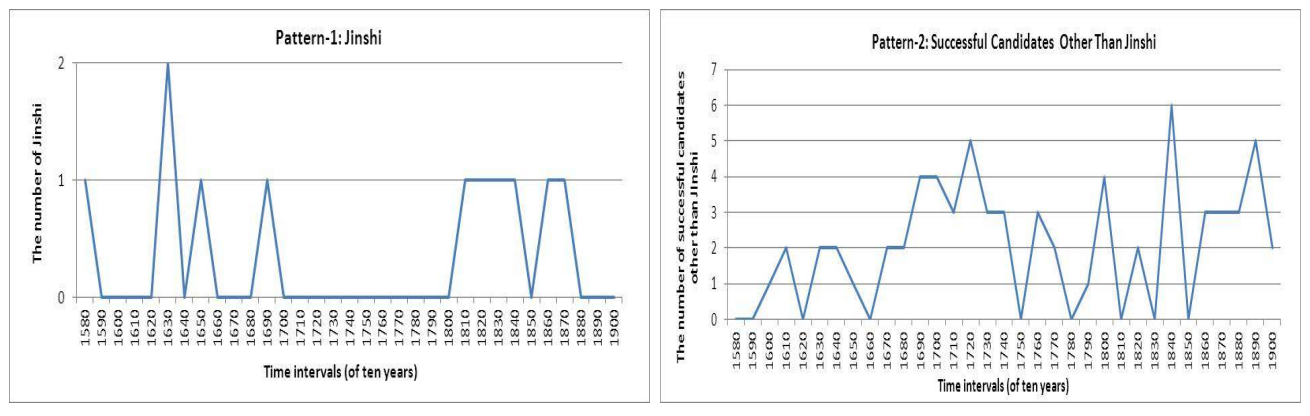

Fig. 4. Successful candidate patterns observed along the $\mathrm{Y}$ family line.
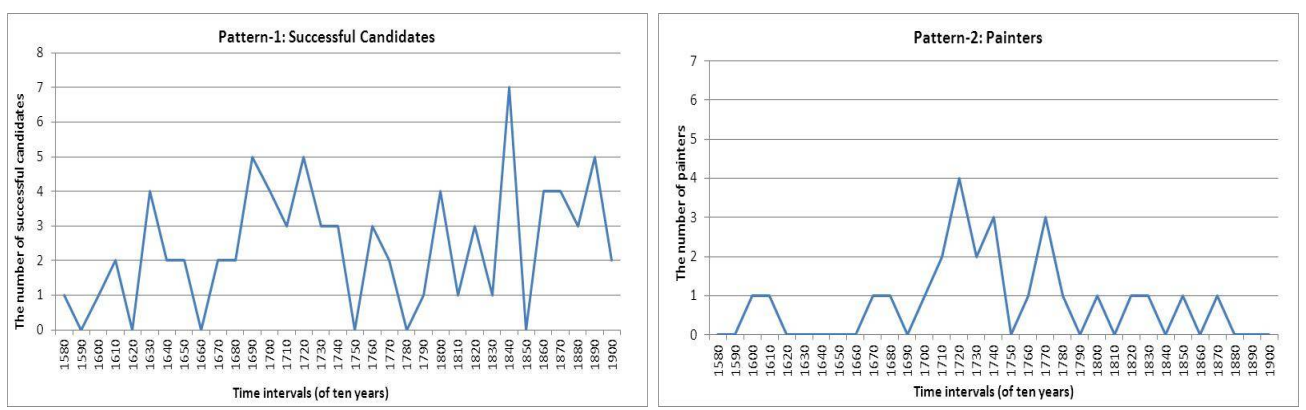

Fig. 5. Successful candidate pattern and painter pattern observed along the Y family line.

As shown in Fig.4, successful candidate patterns at different scales mean that (1) successful candidates who passed the final examination and received titles as Jinshi continued to be produced in the time series, and (2) successful candidates who received titles as Gongshi, Juren, Gongsheng, Jiansheng and Shengyuan continued to be produced in the time series. While in Fig.5, successful candidate pattern and painter pattern with different types refer to (1) successful candidates who received titles as Jinshi, Gongshi, Juren, Gongsheng, Jiansheng or Shengyuan are continued to produce in the time series, and (2) drawing was kept as a traditional life-style in the descendants and painters continued to be produced in the time series.

In this study, we employ multiple patterns observed at different scales and with 
different types to guide the model design and also test alternative assumptions as family strategies.

\section{Model Description with ODD Protocol}

The model description follows the ODD (Overview, Design concepts, Details) protocol for describing individual- and agent-based models [4] [5].

\subsection{Purpose}

The agent-based model is designed to investigate the role of parental relationships and intergenerational reproduction of cultural capital to understand the long-term professional success of an elite family line during the Ming and Qing dynasties in imperial China. The purpose of this case study is to find successful family strategies sustaining a success in the civil service examination in imperial China. We also focus on methodology: the pattern-oriented inverse simulation (POIS) method, where multiple patterns observed from the real system are employed to guide the model design and test alternative assumptions as family strategies, and genetic algorithm based inverse techniques are applied to fit the simulation output with actual data grouped in time intervals as patterns.

\subsection{Entities, state variables, and scales}

The model includes two types of entities, described in Table 1: 1) the school entity represents an influence from school education, and 2) family member entities are individuals of the $\mathrm{Y}$ family line, distinguished by integers from 1 to 1237 as their identifications. State variables are explained in Table 2:1) links between family members are the family tree of the clan, given by elements of the adjacency matrix, 2) attributes of family members mean successful degrees in the examinations, painters, poetry, birth year, etc, given by elements of the attribute matrix, 3) Inborn characteristics of cultural capital, especially, 4) the successful candidate pattern made us introduce the knowledge capital degree variable of the family member entity, where knowledge capital means official linguistic ability required in the examination system, and the painter pattern made us introduce the artistic capital degree variable, which refers to an individual's skill in drawing, 5) influence rates between two types of cultural capital,6),7) and 8) cultural transmission rate describes a discount rate with which cultural capital transmits from school to children, and from family members to children.

In the historical simulation model, cultural capital will be reproduced by different family strategies, and the variables which characterized the strategies determine the result of cultural capital reproduction along with the family line. At the same time, they have common vaiables $\left(m_{0}, m_{1}, m_{2}, m_{3}, m_{4}, m_{5}, m_{6}, r_{a}, r_{k}\right)$ to all the family members, which are described as follows:

- Who transmits cultural capitals (the great grandfather, the grandfather, the father, the mother, the uncle or the aunt $(i))$ ? 
Table 1. Entities and descriptions.

\begin{tabular}{ccc}
\hline Entities & Description & Entities name in the model \\
\hline $\begin{array}{c}\text { School } \\
\text { Family members }\end{array}$ & $\begin{array}{c}\text { Environmental factor as school education } \\
\text { Individuals of the Y family line }\end{array}$ & Id (integers from 1 to 1237) \\
\hline
\end{tabular}

Table 2. State variables and descriptions.

\begin{tabular}{|c|c|c|}
\hline State variables & Description & Variables name in the model \\
\hline Links between family members & Family tree in the clan & Adjacency matrix \\
\hline Attributes of family members & The attributes of individuals & Attribute matrix \\
\hline Inborn characteristics & $\begin{array}{l}\text { Inborn characteristics of } \\
\text { cultural capitals of the child }\end{array}$ & $p s_{c}^{k}, p s_{c}^{a}$ \\
\hline Cultural capital degree & $\begin{array}{l}\text { The level of cultural } \\
\text { capital of the family members }\end{array}$ & $c l_{i}^{k}, c l_{i}^{a}$ \\
\hline Influence rate & $\begin{array}{l}\text { Influence rate between } \\
\text { two types of cultural capitals }\end{array}$ & $r_{a}, r_{k}$ \\
\hline $\begin{array}{l}\text { Transmission rate of } \\
\text { school education }\end{array}$ & $\begin{array}{l}\text { Cultural capital transmission rate } \\
\text { from school to the child }\end{array}$ & $m_{0}$ \\
\hline $\begin{array}{l}\text { Transmission rates from } \\
\text { family members }\end{array}$ & $\begin{array}{l}\text { Cultural capital transmission rate } \\
\text { from family members to the child } \\
\text { Alternative assumptions as "family }\end{array}$ & $\begin{array}{l}m_{1}, m_{2} \\
m_{3}, m_{5}\end{array}$ \\
\hline $\begin{array}{l}\text { Transmission rates from } \\
\text { mother and aunt } \\
\text { (Women's role) }\end{array}$ & $\begin{array}{l}\text { Alternative assumptions as "tamily } \\
\text { follows strategy in educating } \\
\text { child with women's role" } \\
\text { and "family follows strategy in educating } \\
\text { child without women's role" }\end{array}$ & $m_{4}, m_{6}$ \\
\hline
\end{tabular}

- Degree of effect by school education (cultural transmission rate from school education to the child $\left.\left(m_{0}\right)\right)$.

- Degree of effect by family circumstances (cultural transmission rate from the great grandfather, the grandfather, the father, the uncle to the $\left.\operatorname{child}\left(m_{1}, m_{2}, m_{3}, m_{5}\right)\right)$.

- Degree of effect by women's role (cultural transmission rate from the mother's birth family $\left(m_{4}\right)$ and from the aunt's married family $\left(m_{6}\right)$ ).

- Influence rate from knowledge cultural capital to artistic cultural capital $\left(r_{k}\right)$.

- Influence rate from artistic cultural capital to knowledge cultural capital $\left(r_{a}\right)$.

\subsection{Process overview and scheduling}

Each individual accumulates his cultural capital from school education and family members. The following pseudo-code describes the process and scheduling of cultural capital transmissions in agent-based model (Fig.6). A simulation cycle is defined as one execution of cultural transmission along the family tree through each individual.

The agent model is implemented through POIS, we give the pseudo-code of POIS in the following(Fig.7): 


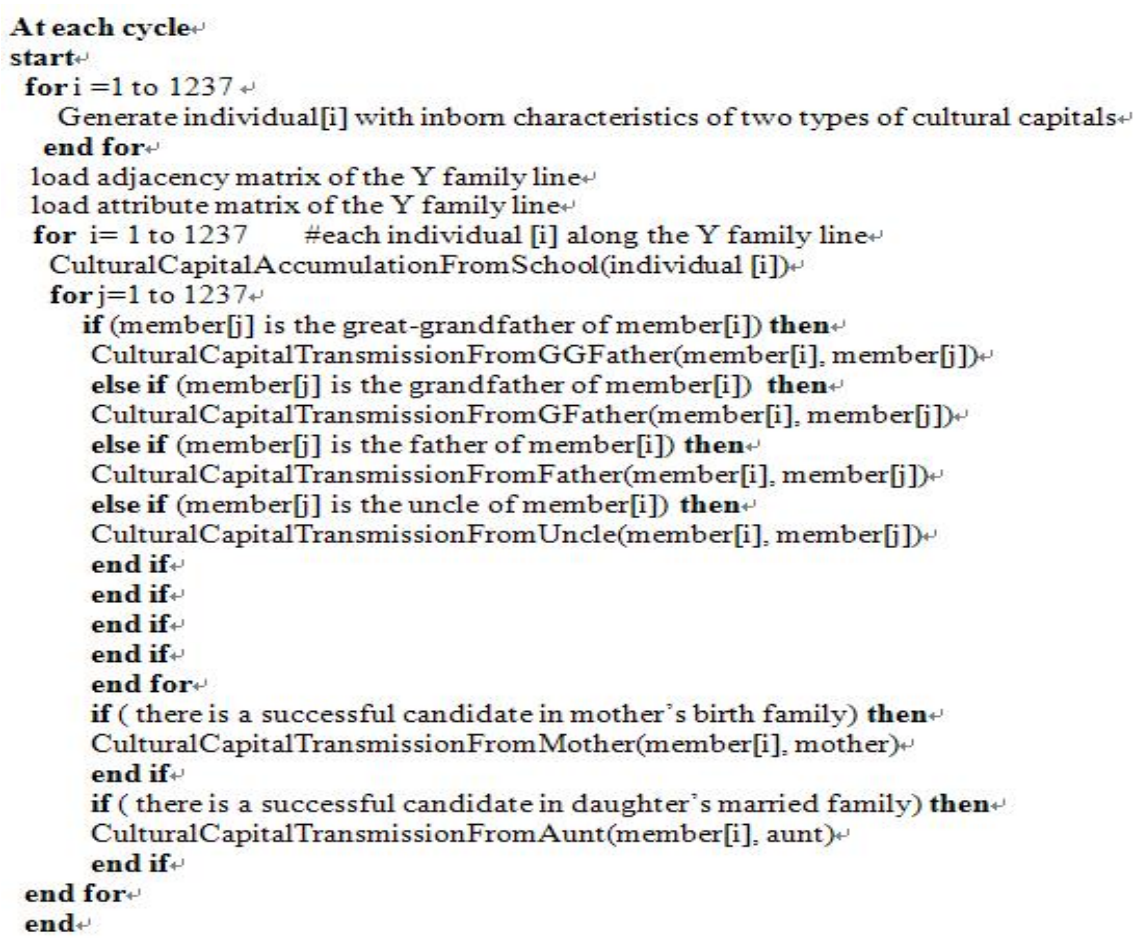

Fig. 6. Pseudo-code of the agent simulation model.

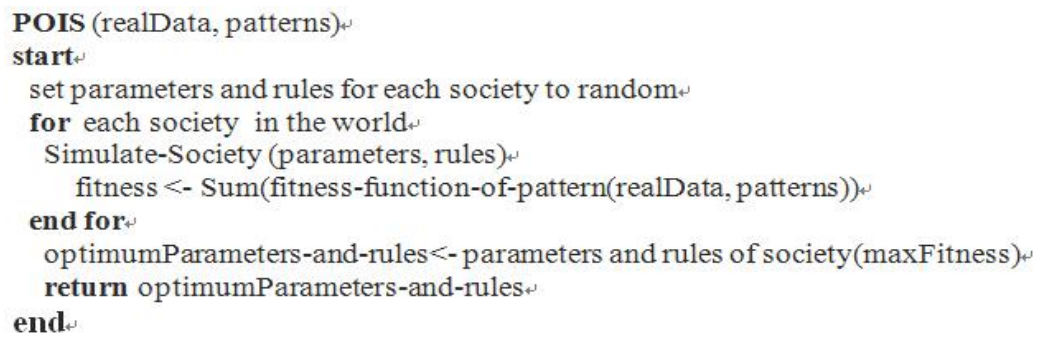

Fig. 7. Pseudo-code of the POIS method.

\subsection{Design concepts}

Basicprinciples. The general concepts underlying the model's design are cultural capital, which are addressed based on Bourdieu's theory of cultural capital[1]. Bourdieu defined cultural capital as the coming together of tangible and intangible property related to culture in the broad sense of the term. In the category, he defined the variety of knowledge, accomplishment, expertise, liking and sensitivity that each 
individual has accumulated through his/her family circumstances and school education as physicalized cultural capital. The second concept designed in the model are cultural transmission process, which comes from Claude's theories on elementary structures of kinship[2]. In the theory, cultural transmission process is mostly happen from the immediate paternal family roles to the descendants. The third concept designed in the model is women's role in educating child, defined from Ko, Mann and Ueno's theories on marriage system, women and culture in Chinese society $[8$, 10, 11, 19].In[10], Mann stated that the daughters' education could provide the potential to successfully manage her married family. Ko discussed that, highly educated wives or mothers were considered to have good effects on the education of the children, the activation of cultural activities such as poetry and drawing, and the improvement of morals[8]. In further, Mann pointed out that well-educated women were highly evaluated in their regional societies and the marriage alliances[11]. Ueno has stated that both families kept their relationship through women's marriage in educating child[19].

Three assumptions addressed following the corresponding theories are: (1) Cultural capitals are classified into knowledge cultural capital and artistic cultural capital; (2) Cultural capital is mainly transmitted from the immediate paternal family roles such as the grandfather, the father and the uncle; (3)Cultural capital can also pass on from the mother to the son, and from the aunt to the nephew through women's marriage. In the way, the alternative hypotheses about family strategy in this model are "family follows strategy in educating child with women's role" or "family follows strategy in educating child without women's role".

Objectives. The objective function of the model is defined as the minimization of sum-of-squares errors between simulated data and actual data.

Learning. The family taken as a whole system learns good family strategies in civil service examination in imperial China, which indicates family norms in successful cultural transmission activities.

Stochasticity. When the simulation starts, the inborn characteristics are given to each individual(agent) as a random real number from 0 to 0.3 .

Observation. The data collected from the ABM is the number of successful candidates and painters, which is grouped in time intervals as patterns.

\subsection{Initialization}

At the initial stage, the model randomly generates 1,237 individuals (agents) to represent individuals of the family tree, with their inborn characteristics of knowledge cultural capital and artistic cultural capital (random real numbers from 0 to 0.3 ). We set the linkages between family members according to those "1" values in the adjacency matrix. 


\subsection{Submodels}

In detail, two sub models are presented to describe the operation of cultural capital transmission processes described in 'Process overview and scheduling'. We defined two equations in the following: Eq. (1) calculates the knowledge cultural capital of the child, and Eq. (2) calculates the artistic cultural capital of the child.

$$
\begin{aligned}
& c l_{c}^{k}=m_{0} \cdot\left(p s_{c}^{k}+r_{a} \cdot p s_{c}^{a}\right)+\sum_{i=1}^{n} m_{i} \cdot\left(c l_{i}^{k} \cdot p s_{c}^{k}+r_{a} \cdot c l_{i}^{a} \cdot p s_{c}^{a}\right), \\
& c l_{c}^{a}=m_{0} \cdot\left(p s_{c}^{a}+r_{k} \cdot p s_{c}^{k}\right)+\sum_{i=1}^{n} m_{i} \cdot\left(c l_{i}^{a} \cdot p s_{c}^{a}+r_{k} \cdot c l_{i}^{k} \cdot p s_{c}^{k}\right) .
\end{aligned}
$$

Where $k$ and $a$ represents knowledge capital and artistic capital, respectively. Other variables are explained in 'Entities, State Variables and Scales'. The first term of Eq. (1) and (2) model cultural transmission process from school to children, and their second terms model cultural transmission process from family members (such as great grandfathers, grandfathers, fathers, mothers, uncles and aunts) to children.

\section{Agent Simulation through POIS}

The agent simulation is implemented through POIS, using GA-based validations, illustrated in Fig.8. Parameter sets which indicate different family strategies are operated on the family tree through agent-based simulation. By agent-based simulation, we obtained simulated cultural capital of all the agents. While real cultural capital is obtained from the attribute matrix, measured by the value of " 0.5 " or " 0 " (In this study, we used the same threshold "0.5" to represent a qualified statue given by passing the examination, the real value itself has no special meaning, which can be replaced by any real value other than zero). Both simulated and actual data are grouped in time intervals (of each ten year period from the birth years). They appear as patterns. The repetition of so many multi-agent simulations (MASs) evolves the simulated multiple patterns (output data grouped in time intervals) to fit to the observed patterns (actual data grouped in time intervals).

As described in Section 3, we observed (1)successful candidate patterns at different scales, and (2) successful candidate pattern and painter pattern with different types. These patterns will guide the model design and test alternative assumptions as family strategies.

When employing successful candidate patterns at different scales in POIS, the evaluation rules are (1) if the value of simulated cultural capital is equal to or greater than 0.5 , then the agent is evaluated as a Jinshi, (2) if the simulated value is between 0.3 and 0.5 , then the agent is evaluated as a successful candidate other than Jinshi, (3) or else if the simulated value is smaller than 0.3 , then the agent is not a successful candidate on the examinations. 


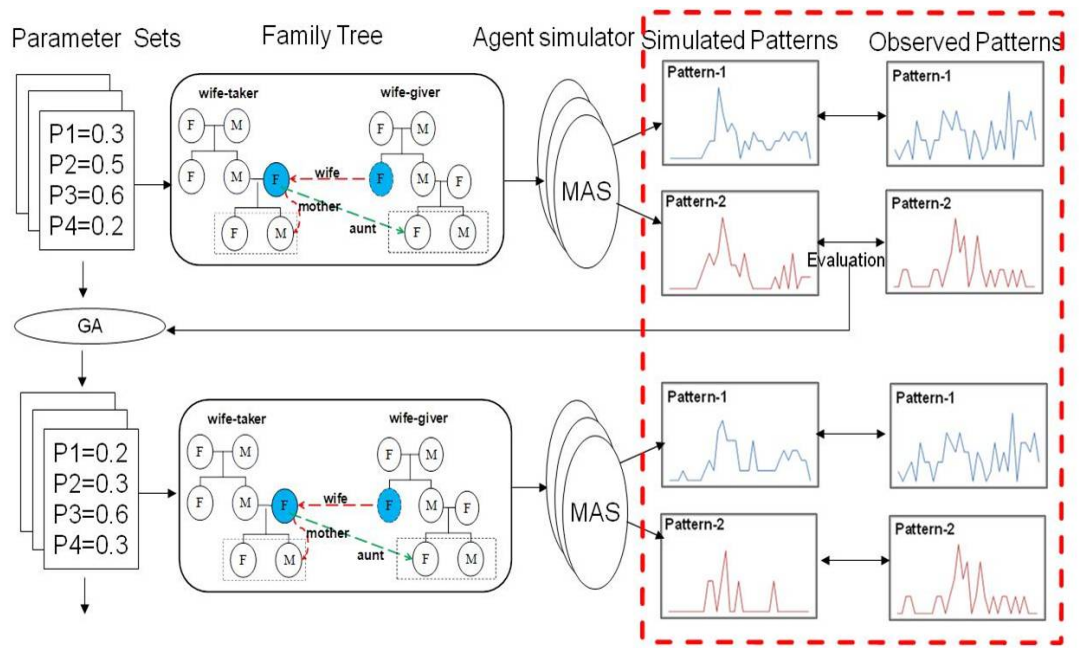

Fig. 8. Agent simulation through POIS.

When employing successful candidate pattern and painter pattern with different types in POIS, the evaluation rules are (1) if the value of simulated cultural capital is equal to or greater than 0.5 , the agent is evaluated as a successful candidate or a painter, depending on the type of cultural capital; (2) or else if the simulated value is smaller than 0.5 , the agent is neither a successful candidate nor a painter.

We finally obtained a model structure which reproduces the observed multiple patterns similar to the historical records of the $\mathrm{Y}$ family line. By analyzing the parameters of this model, we could estimate the strategies of the $\mathrm{Y}$ family which produced more successful candidates in civil service examinations in imperial China.

\section{Simulation Experiments}

We conducted two groups of simulation experiments to examine the practicability of the POIS in historical simulation domain. All simulation experiments are implemented through the POIS method by utilizing the SOMAS framework, which is developed by Yang et al. for easy execution of multiple runs of ABS experiments [22]. We summarized the methods of simulation experiments in Table 3.

The first group of simulation experiments tests how multiple patterns works to guide the design of model structure. Among this group, simulation experiment 1 employs a single pattern that (1) successful candidates who received qualified titles as Jinshi, Gongshi, Juren, Gongsheng, Jiansheng and Shengyuan were sustained in the time series, and (2) drawing was kept as a traditional life style in the descendants and painters were sustained in the time series respectively to reproduce patterns observed in the Y family line. The simulation setting corresponds to the IS with a single pattern used in [9]. 
Table 3. Summary of the methods of simulation experiments.

\begin{tabular}{lcc}
\hline Group No. & Simulation experiment No. & Methods \\
\hline \multirow{2}{*}{ Group 1 } & Simulation experiment 1 & Pimulation experiment 2 \\
& Simulation experiment 3 & POIS with two patterns at different scales \\
& POIS with two patterns with different types \\
\hline Group 2 & Simulation experiment 4 & POIS without women's role \\
\hline
\end{tabular}

In simulation experiment 2, there were two patterns of successful candidates at different scales where (1) successful candidates who passed the final examination and received titles as Jinshi were sustained in the time series, and (2) successful candidates who received titles as Gongshi, Juren, Gongsheng, Jiansheng or Shengyuan were sustained in the time series are employed to reproduce patterns observed in the Y family line.

In simulation experiment 3, successful candidate pattern and painter pattern with different types are employed to reproduce patterns observed in the $\mathrm{Y}$ family line. This is the method of POIS with multiple patterns with different types which we propose in this study.

The second group of simulation experiments test and contrast alternative assumptions of women's role in educating their children: "family follows strategies in educating child with women's role" or "family follows strategies in educating child without women's role". The alternative assumptions are implemented in the agentbased model by two variables as the transmission rates from the mother and the aunt to the children, and tested by how well they reproduced identified multiple patterns.

All simulation experiments execute the cultural capital reproduction processes based on Eq. (1) and Eq. (2), by operating parameter sets as school and family members. We employed Real-coded GA(RCGA) for optimizations in POIS. RCGA is a type of GA which employs real numbers to represent the variables or parameters of the model. The employed algorithms for GA operations are the same as our previous work in [21].

All simulation experiments set the configurations of GA as follows: selection by best and rank-based roulette, the number of times of crossover in MGG [15] is 100, the number of the child populations per each generation of MGG is 200, alpha of UNDX [12] is 0.5 and beta of UNDX is 0.35 , the number of the initial populations is 50 , and the maximum generation is set at 2, 000, where the values of alpha and beta in UNDX were set according to the suggestion in [12].

The measure of the model is set by the value of sum-of squares error (SSE), in order to assure the consistent of patterns and minimize the errors between the actual data and the simulated data. Table 4 shows the average value of the SSE over ten trials obtained by simulation experiments of the first group. It is obvious that simulation experiment 3 successfully found the smallest SSE when compared with 
the other two simulation experiments, by employing successful candidate pattern and painter pattern with different types, which is the proposed method of POIS with multiple patterns observed with different types for modeling.

Table 4. Summary of SSEs.

\begin{tabular}{|c|c|c|c|}
\hline Simulation experiment No. & Employed patterns & SSEs of patterns & sum of SSEs \\
\hline \multirow[t]{2}{*}{ Simulation experiment 1} & successful candidate pattern & $\begin{array}{c}\text { successful candidate: } 26.75 \\
\text { painter: } 24.25\end{array}$ & 51.00 \\
\hline & painter pattern & $\begin{array}{c}\text { successful candidate: } 59.25 \\
\text { painter: } 9.50\end{array}$ & 68.75 \\
\hline Simulation experiment 2 & $\begin{array}{l}\text { successful candidate pattern } \\
\text { at different scales }\end{array}$ & $\begin{array}{c}\text { Jinshi: } 3.25 \\
\text { Other successful candidate: } 40.75 \\
\text { painter: } 13.5\end{array}$ & 57.50 \\
\hline Simulation experiment 3 & $\begin{array}{l}\text { successful candidate pattern } \\
\text { and painter pattern } \\
\text { (with women's role) }\end{array}$ & $\begin{array}{c}\text { successful candidate: } 25 \\
\text { painter: } 10.25\end{array}$ & 35.25 \\
\hline Simulation experiment 4 & $\begin{array}{l}\text { successful candidate pattern } \\
\text { and painter pattern } \\
\text { (without women's role) }\end{array}$ & $\begin{array}{c}\text { successful candidate: } 35.75 \\
\text { painter: } 13.00\end{array}$ & 48.75 \\
\hline
\end{tabular}

Because the model structure obtained by employing two patterns with different types in the simulation experiment 3 has the minimal sum-of squares error between the actual historical data and the simulated data, we therefore conducted the second group of simulation experiments (simulation experiment 4) based on the parameter settings of the simulation experiment 3. Consequently, we removed two parameters as the transmission rates from the mother and the aunt from the simulation experiment 3 , in order to conduct the simulation experiment 4 to test and contrast the theories of women's role in educating their children. The SSE over ten trials obtained in the simulation experiment 4 is 48.75 (SSE of pattern- $1=35.75$ and SSE of pattern-2 =13.00), larger than in the simulation experiment 3(Table 4). This indicates that the influences from mothers and aunts really exist in the cultural capital reproduction process, which supports the theories of women's role in educating their children [8].

Furthermore, we summarized the average parameter values obtained by simulation experiment 3 in Table 5.

The reproduced patterns obtained in the simulation experiment 3 were given by Fig.9. The simulated data are grouped in time intervals of each ten year period from the individual's birth years, which appears as patterns in the time series.

\section{Discussions}

In the model, we focused on family strategies(norms) behind the whole family system in conveying cultural capitals to the descendants, rather than individual-based 
Table 5. Average parameter values obtained by experiment 3 .

\begin{tabular}{ccc}
\hline Parameters and their descriptions & $\begin{array}{c}\text { Transmission rate } \\
\text { (Average of ten trials) }\end{array}$ & Standard deviation \\
\hline Cultural transmission rate from school $\left(m_{0}\right)$ & 0.18 & 0.06 \\
Cultural transmission rate from great grandfather $\left(m_{1}\right)$ & 0.08 & 0.04 \\
Cultural transmission rate from gandfather $\left(m_{2}\right)$ & 0.17 & 0.04 \\
Cultural transmission rate from father $\left(m_{3}\right)$ & 0.16 & 0.05 \\
Cultural transmission rate from mother $\left(m_{4}\right)$ & 0.13 & 0.01 \\
Cultural transmission rate from uncle $\left(m_{5}\right)$ & 0.06 & 0.01 \\
Cultural transmission rate from aunt $\left(m_{6}\right)$ & 0.21 & 0.03 \\
Influence rate from knowledge capital to artistic capital $\left(r_{a}\right)$ & 0.71 & 0.04 \\
Influence rate from artistic capital to knowledge capital $\left(r_{k}\right)$ & 0.29 & 0.04 \\
\hline
\end{tabular}
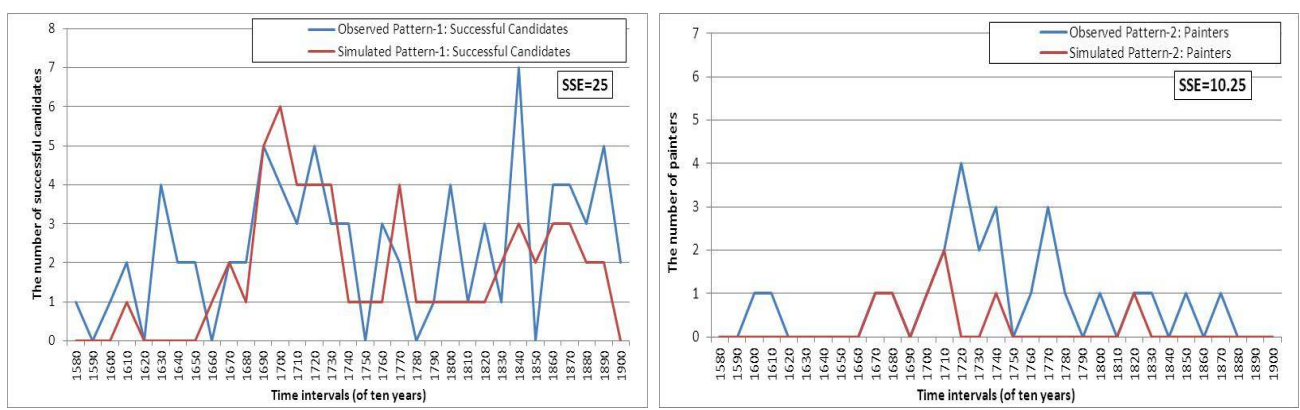

Fig. 9. Reproduced patterns by employing two patterns with different types.

decision making. As simulation experiment 3 has the minimal sum-of-squares error $(\mathrm{SSE}=35.25)$ between the actual historical data and the simulated data, we selected the results as the optimal solution for successful family strategies for cultural capital reproduction of the $\mathrm{Y}$ family line. Based on the results in Table 5, we discovered that both school and family members played important roles in the social cultural capital reproduction process. Especially, the role of the grandfather is greater than the father in educating the child and the great grandfather passes on little cultural capital to their great grandsons. The optimal solution has also proved the important role of the mother and the aunt in educating their children. Besides, although the influences from uncles are relatively lower, their influences still exist in the cultural transmission process. Furthermore, artistic ability has positive influence for increasing knowledge capital $\left(r_{a}=0.71\right)$ while the inverse influence is relatively small $\left(r_{k}=0.29\right)$.

We discussed the simulation results as follows: (1) The failure of simulation experiment 1 is caused by only one pattern employed in the simulation. You can only observe the phenomenon of activities in the $\mathrm{Y}$ family using a single pattern, but it is not sufficient to see real phenomenon represented by multiple patterns with 
different types.

(2) The failure of simulation experiment 2 exists in : 1) the final examination was too difficult and only a small number of Jinshi (one or two persons per each time interval) was limited to be used to reproduce the patterns, 2) only the successful candidate patterns at different scales were not enough to see the pattern of the artistic cultural type.

(3) The simulation experiment 3 successfully reproduced two types of patterns near the real trend in Fig.5. Multiple patterns with different types are therefore proved important and useful to analyze the internal organizations of the $\mathrm{Y}$ family line, while only a single pattern or multiple patterns observed at different scales do not work well.

(4) The simulation experiment 4 proved the important role of the mothers and the aunts in educating their child. We therefore verified the assumption as "family follows strategies in educating the child with women's role". It indicates such strategies to help rebuild the family fortunes: a marriage with girls from "good families" or girls married to "good families". However, the same woman may act as a mother to her son or an aunt to her nephew under a big family system in imperial China. So do the roles of fathers and uncles. For the reason, it is difficult for us to explain the precise influence rate of each role.

Based on the above analysis, the main findings are summarized as follows: (1)school education helps to increase the child's cultural capital, (2)grandfathers play an important role in educating grandsons, (3) both mothers from the wifetaker side and aunts from the wife-giver side pass on cultural capital to the child, which have proved a reciprocal relationship between family systems in cultural capital reproduction activities, and (4) a combined influence of grandfathers, fathers, mothers, aunts and uncles are important to maintain a successful family norm in cultural capital reproduction in imperial China.

The result of such screenings has been confirmed by matching them against the actual records and statistical analysis, and it has successfully reproduced multiple patterns observed with different types of the $\mathrm{Y}$ family line. It may be possible that these facts are the customs which were transmitted as the family strategies (norms) over generations.

\section{Concluding Remarks}

This paper has proposed a pattern-oriented inverse simulation (POIS) method to analyze social problems based on the recent advances of the POM strategy and the IS method in ACSs. We have applied POIS to a historical simulation domain, in order to analyze a particular family line which produced more successful candidates in civil service examination in imperial China. Our previous simulation model on POM only employed a single pattern to analyze the internal organization of the cultural capital reproduction process of the $\mathrm{Y}$ family line, which failed to explain multiple patterns observed with different types in the $\mathrm{Y}$ family line. As an extension, 
this study employs the IS method to POM, which selects the appropriate internal structure of real family systems by operating the simulation model to fit two patterns observed with different types through inverse simulation techniques. POIS has come to be able to optimize the combination of appropriate patterns and the selection of the internal structure which are only trial and error in POM method.

We have employed multiple patterns observed with different types of the $\mathrm{Y}$ family line to optimize the parameters of family strategies. The simulation results have proved the importance and usefulness of multiple patterns observed with different types to find the internal organizations of social systems, while a single pattern or only multiple patterns at different scales of the same type do not perform well. The best values of parameters obtained by POIS have successfully reproduced two patterns observed with different types in the particular family line (the Y family line).

From intensive simulation experiments by POIS, we have obtained a model structure with its parameters indicating successful family strategies in civil service examination in imperial China. The simulation results discovered family strategies that sustained success in the examination systems in imperial China. This relates to important aspects of sociological theories on the role of cultural capital to transmit social inequality intergenerationally. This case study also proved that the new methodology of POIS can improve our current practices for systematically exploring simulation parameter space and fit model output with actual data. Our conclusion is that we should develop agent-based models using multiple patterns observed with different types, so that we are able to ground the agent-based models to real world problems.

\section{References}

[1] Bourdieu, P., Distinction: A Social Critique of the Judgment of Taste (Harvard University Press, 1979).

[2] Claude, L. S., The elementary structures of kinship. trans. by James Harle Bell, John Richard von Sturmer and Rodney Needham (London: Eyre \& Spottiswoode, 1969).

[3] Elman, B. A., A Cultural History of Civil Examinations in Late Imperial China (Berkeley: University of Californian Press, 2000).

[4] Grimm, V., Berger, D. L., U.and Deangelis, Polhill, J. G., and Giske, S. F., J.and Railsback, The odd protocol: A review and first update, Ecological Modelling 221 (2010) 2760-2768.

[5] Grimm, V., Berger, F., U.and Bastiansen, Eliassen, S., Ginot, J. G.-C. J., V.and Giske, Grand, S. H.-G. H. A., T.and Heinz, Jepsen, J., Jorgensen, C., Mooij, W., Muller, B., Pe' er, G., Piou, C., Railsback, S., Robbins, A., Robbins, M., Rossmanith, E., Ruger, N., Strand, E., Souissi, S., Stillman, R., Vabo, R., Visser, U., and DeAngelis, D., A standard protocol for describing individual-based and agent-based models, Ecological Modelling 198 (2006) 115-126.

[6] Grimm, V., Revilla, E., Berger, U., Jeltsch, F., Mooij, W., Railsback, S., Thulke, H., Weiner, J., Wiegand, T., and DeAngelis, D., Pattern-oriented modeling of agentbased complex systems: Lessons from ecology, Science 310 (2005) 987-991.

[7] Janssen, M. A., Radtke, N. P., and Lee, A., Pattern-oriented modeling of commons 
dilemma experiments, Adaptive Behavior 17 (2009) 508-523.

[8] Ko, D., Teachers of the Inner Chambers, Women and Culture in Seventeenth-Century China (Stanford University Press, 1994).

[9] Kurahashi, S. and Terano, T., Historical simulation: A study of civil service examinations, family line, and cultural capital in china, in the 4 th Conference of the European Social Simulation Association (ESSA'07) (2007).

[10] Mann, S., Grooming a daughter for marriage: Brides and wives in the mid-ching period, In Watson $R S$ and Ebrey $P B$ (Eds.), Marriage and Inequality in Chinese Society (University of California Press, 1991).

[11] Mann, S., Precious Records, Women in China's Long Eighteenth Century (Stanford University Press, 1997).

[12] Ono, I., Kita, H., and Kobayashi, S., A Real-Coded Genetic Algorithm Using the Unimodal Normal Distribution Crossover (Springer, 2002).

[13] Rademacher, C., Neuert, C., Grundmann, V., Wissel, C., and Grimm, V., Reconstructing spatiotemporal dynamics of central european natural beech forests: the rule-based forest model 3EFORE, Forest Ecology and Management 194 (2004) 349368.

[14] Rouchier, J., Bousquet, F., Requier-Desjardins, M., and Antona, M., A multi-agent model for describing transhumance in north cameroon: Comparison of different rationality to develop a routine, Journal of Economic Dynamics and Control 25 (2001) $527-559$

[15] Sato, H., Ono, I., and Kobayashi, S., A new generation alternation model of genetic algorithms and its assessment, Journal of the Japanese Society for Artificial Intelligence 12 (1997) 734-735.

[16] Terano, T., Exploring the vast parameter space of multi-agent based simulation, in Multi-Agent-Based Simulation (MABS 2006), eds. Antunes, L. and Takadama, K. (Springer, 2007), pp. 1-14, lecture Notes in Computer Science 4442.

[17] Terano, T. and Kurahashi, S., Inverse simulation: Genetic-algorithm based approach to analyzing emergent phenomena, in the International Workshop on Emergent Synthesis (IWES'99) (1999), pp. 271-276.

[18] Terano, T., Kurahashi, S., and Minami, U., TRURL: Artificial world for social interaction studies, in the 6th International Conference on Artificial Life (ALIFE VI) (1998), pp. 326-335.

[19] Ueno, H., Relative of Han People in Taiwan (in Japanese) (Fukyo-sha, 2000).

[20] Wiegand, T., Revilla, E., and Knauer, F., Dealing with uncertainty in spatially explicit population models, Biodiversity and Conservation 13 (2004) 53-78.

[21] Yang, C., Kurahashi, S., Kurahashi, K., Ono, I., and Terano, T., Agent-based simulation on women's role in a family line on civil service examination in Chinese History, Journal of Artificial Societies and Social Simulation 12 (2009).

[22] Yang, C., Ono, I., Kurahashi, S., and Terano, T., SOMAS: an integrated framework for social simulators on a grid environment, in the 43rd Systems Engineering Sectional Meeting (Social Simulation and Service System Symposium) (2010), pp. 139-144. 